$10,11,05$

\title{
Критические свойства антиферромагнитной слоистой модели Изинга на кубической решетке с конкурирующими взаимодействиями
}

\author{
(ㄱ А.К. Муртазаев ${ }^{1,2}$, М.К. Рамазанов ${ }^{1, \uparrow}$ \\ ${ }^{1}$ Институт фозики им. Х.И. Амирханова ДагНЦ РАН, \\ Махачкала, Россия \\ ${ }^{2}$ Дагестанский государственный университет, \\ Махачкала, Россия \\ ฯ E-mail: sheikh77@mail.ru
}

(Поступила в Редакцию 21 февраля 2017 г.)

На основе репличного алгоритма методом Монте-Карло выполнены исследования критических свойств антиферромагнитной слоистой модели Изинга на кубической решетке с учетом взаимодействий ближайших и следующих за ближайшими соседей. Исследования проведены для соотношений величин обменных взаимодействий следующих и ближайших соседей $r=J_{2} / J_{1}$ в диапазоне значений $0 \leq r \leq 1.0$. В рамках теории конечно-размерного скейлинга рассчитаны статические критические индексы теплоемкости $\alpha$, параметра порядка $\beta$, восприимчивости $\gamma$, радиуса корреляции $v$, а также индекс Фишера $\eta$. Показано, что класс универсальности критического поведения этой модели сохраняется в диапазоне значений $0 \leq r \leq 0.4$. Установлено, что изменение величины взаимодействия следующих ближайших соседей в данной модели в диапазоне $r>0.8$ приводит к тому же классу универсальности критического поведения, что и трехмерная полностью фрустрированная модель Изинга на кубической решетке.

Исследование выполнено при финансовой поддержке РФФИ в рамках научного проекта № 16-02-00214-а.

DOI: $10.21883 /$ FTT.2017.09.44854.048

\section{1. Введение}

Проблема исследования фазовых переходов (ФП) и критических свойств в спиновых системах с конкурирующим обменным взаимодействием является одной из центральных в современной физике конденсированного состояния [1-3]. Конкуренция обменного взаимодействия может привести к фрустрации.

Известно, что фрустрированные системы (ФС) во многом проявляют свойства, отличные от соответствующих нефрустрированных систем. Это отличие выражается в богатом разнообразии фаз и ФП, что обусловлено сильным вырождением и высокой чувствительностью ФС к различного рода возмущающим взаимодействиям. Кроме того, можно отметить проблемы связанные с определением характера ФП, с особенностями и факторами влияющими на формирование классов универсальности магнитного и кирального критического поведения фрустрированных спиновых систем и др. [4,5].

Одним из наиболее интенсивно исследуемых в последние годы фрустрированных моделей является двумерная модель Изинга на квадратной решетке с учетом взаимодействий следующих ближайших соседей [5-13]. Эта модель изучена достаточно хорошо и почти все ее свойства известны. ФП и критические свойства этой модели для трехмерного случая практически не исследованы. При учете антиферромагнитных взаимодействий следующих ближайших соседей в классической трехмерной модели Изинга сопровождается вырождением основного состояния и появлением различных фаз и ФП. Кроме того, учет взаимодействия следующих ближайших со- седей может также влиять на критическое поведение модели [4].

В работах $[14,15]$ нами были проведены исследования ФП и критических свойств антиферромагнитной слоистой модели Изинга на кубической решетке с учетом взаимодействий следующих ближайших соседей внутри слоев решетки. Эта модель является частным случаем модели исследуемой в работах [16,17], когда взаимодействие следующих ближайших соседей между слоями равно нулю. В работе [14] был рассмотрен случай, когда $r=1.0\left(J_{1}\right.$ и $J_{2}-$ константы обменного взаимодействия ближайших и следующих за ближайшими соседей соответственно), где $r=J_{2} / J_{1}-$ величина взаимодействия следующих за ближайшими соседей. Было показано, что для случая $r=1.0$ в исследуемой модели переход из суперантиферромагнитной фазы в парамагнитную реализуется как ФП второго рода. Рассчитаны все основные статические критические индексы для случая $r=1.0$. Было показано, что эта модель принадлежит к тому же классу универсальности критического поведения, что и трехмерная полностью фрустрированная модель Изинга. В работе [15] было показано, что в диапазоне $0 \leq r \leq 0.5$, а также для значения $r=0.9$ наблюдается ФП второго рода, а в диапазоне $0.6 \leq r \leq 0.8$ наблюдается ФП первого рода.

В настоящей работе нами предпринята попытка на основе репличного алгоритма метода Монте-Карло (МК) изучить критическое поведение и вычислить полный набор статических критических индексов антиферромагнитной слоистой модели Изинга на кубической решетке 
с учетом взаимодействий ближайших и следующих за ближайшими соседей внутри слоев решетки в диапазоне $0 \leq r \leq 0.5$, а также для значений $r=0.9$ и $r=1.0$, где наблюдается ФП второго рода $[14,15]$.

Интерес к этой модели обусловлен тем, что многие физические свойства ФС сильно зависят от величины взаимодействия следующих ближайших соседей. Кроме того, антиферромагнитная слоистая модель Изинга на кубической решетке с учетом взаимодействия следующих ближайших соседей до сих пор является малоизученной. Таким образом, исследование этой модели на основе современных методов позволит получить ответ на ряд вопросов, связанных с критическими свойствами фрустрированных спиновых систем.

\section{2. Модель и метод исследования}

Антиферромагнитная модель Изинга на кубической решетке с учетом взаимодействий следующих ближайших соседей описывается следующим гамильтонианом $[14,15]$ :

$$
H=J_{1} \sum_{\langle i, j\rangle}\left(S_{i} \cdot S_{j}\right)+J_{2} \sum_{\langle i, j\rangle}\left(S_{i} \cdot S_{l}\right),
$$

где $S= \pm 1$ - изинговский спин. Решетка состоит из двумерных квадратных слоев, сложенных по ортогональной оси. Первый член в формуле (1) учитывает обменное взаимодействие ближайших соседей, которое берется одинаковым как внутри слоев решетки, так и между слоями $\left(J_{1}>0\right)$, второй - следующих за ближайшими соседей, находящихся в том же слое решетки $\left(J_{2}>0\right)$.

Модель Изинга на кубической решетке при учете антиферромагнитных взаимодействий следующих ближайших соседей внутри слоев решетки становится фрустрированной. Фрустрации в этой модели обусловлены конкуренцией обменных взаимодействий между ближайшими и следующими за ближайшими соседями.

Исследование критических свойств фрустрированных спиновых систем традиционными теоретическими, экспериментальными и численными методами сталкиваются с рядом труднопреодолимых проблем. Это связано с тем, что для таких систем характерна проблема многочисленных долин локальных минимумов энергии. Строго и последовательно на основе микроскопических гамильтонианов такие системы могут быть изучены методами МК [18-24]. Наиболее мощными и эффективными в исследовании ФП и критических явлений в ФС оказались репличные алгоритмы метода МК $[25,26]$. Поэтому в данном исследовании был использован высокоэффективный репличный обменный алгоритм метода МК. Более подробно этот алгоритм описан нами в работе [9].

Расчеты проводились для систем с периодическими граничными условиями и линейными размерами $L \times L \times L=N$, где $L=24-48$ и измеряется в размерах элементарной ячейки. Соотношение обменного взаимодействия следующих и ближайших соседей менялось в интервале $0 \leq r \leq 1.0$. Для вывода системы в состояние термодинамического равновесия отсекался участок длиной $\tau_{0}=4 \cdot 10^{5}$ шагов МК на спин, что в несколько раз больше длины неравновесного участка. Усреднение термодинамических параметров проводилось вдоль марковской цепи длиной до $\tau=500 \tau_{0}$ шагов МК на спин.

\section{3. Результаты моделирования}

Для наблюдения за температурным ходом теплоемкости $C$ и восприимчивости $\chi$ использовались выражения [27]:

$$
\begin{gathered}
C=\left(N K^{2}\right)\left(\left\langle U^{2}\right\rangle-\langle U\rangle^{2}\right), \\
\chi=\left\{\begin{array}{ll}
(N K)\left(\left\langle m^{2}\right\rangle-\langle|m|\rangle^{2}\right), & T<T_{N} \\
(N K)\left\langle m^{2}\right\rangle, & T \geq T_{N}
\end{array},\right.
\end{gathered}
$$

где $K=\left|J_{1}\right| / k_{\mathrm{B}} T, N-$ число частиц, $T_{N}$ - критическая температура, $U-$ внутренняя энергия, $m$ - параметр порядка ( $U$ и $m$ являются нормированными величинами).

Параметр порядка системы $m$ вычислялся с помощью следующих выражений:

$$
\begin{gathered}
m_{\lambda}=\frac{4}{N} \sum_{i \in \lambda}(-1)^{z} S_{i}, \quad \text { где } \lambda=1,2,3,4, \\
m^{a}=\left[m_{1}+m_{2}-\left(m_{3}-m_{4}\right)\right] / 4, \\
m^{b}=\left[m_{1}+m_{4}-\left(m_{2}+m_{3}\right)\right] / 4, \\
m=\sqrt{\left(m^{a}\right)^{2}+\left(m^{b}\right)^{2}},
\end{gathered}
$$

где $m_{1}, m_{2}, m_{3}, m_{4}$ - параметры порядка по подрешеткам, $z$ - номер слоя решетки.

На рис. 1, 2, 3 и 4 представлены температурные зависимости теплоемкости и восприимчивости, полученные при $L=30$ для различных значений $r$ (здесь и далее статистическая погрешность не превышает размеров символ, использованных для построения зависимостей). Отметим, что увеличение значения $r$ в интервале $0 \leq r \leq 0.5$ сопровождается сдвигом максимумов в сторону более низких температур, одновременно с этим наблюдается рост абсолютных значений максимумов как теплоемкости, так и восприимчивости. Рост абсолютных значений максимумов происходит за счет конкуренции ближайших и следующих ближайших соседей. В случае, когда $0.6 \leq r \leq 1.0$, наблюдаем противоположную картину. С увеличением $r$ от 0.6 до 1.0 температура фазового перехода смещается в сторону более высоких температур, при этом наблюдается спад абсолютных значений максимумов теплоемкости и восприимчивости. Увеличение взаимодействия следующих ближайших соседей в этом интервале, приводит к увеличению энергии взаимодействия по модулю, что укрепляет жесткость системы и соответственно повышается температура фазового перехода. 


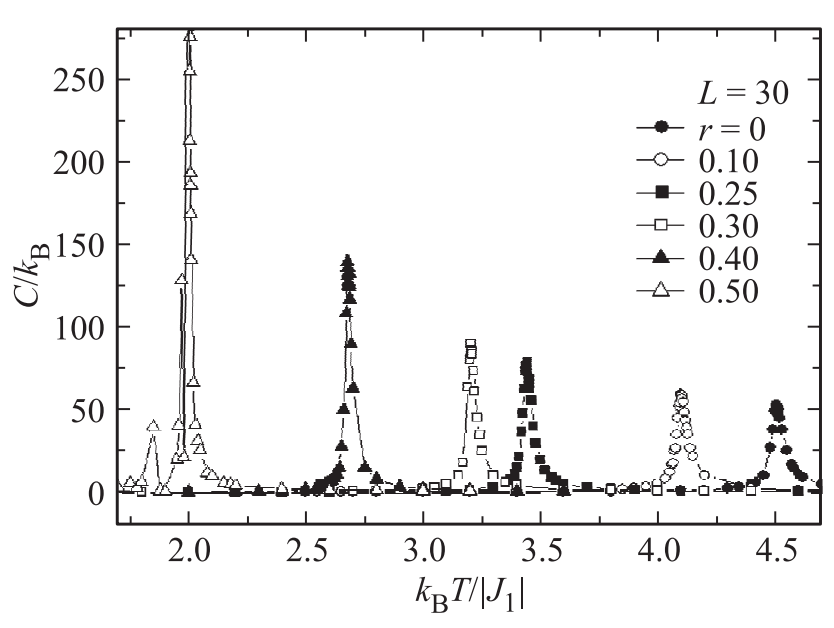

Рис. 1. Зависимость теплоемкости $C / k_{\mathrm{B}}$ от температуры $k_{\mathrm{B}} T /\left|J_{1}\right|$ для различных $r$.

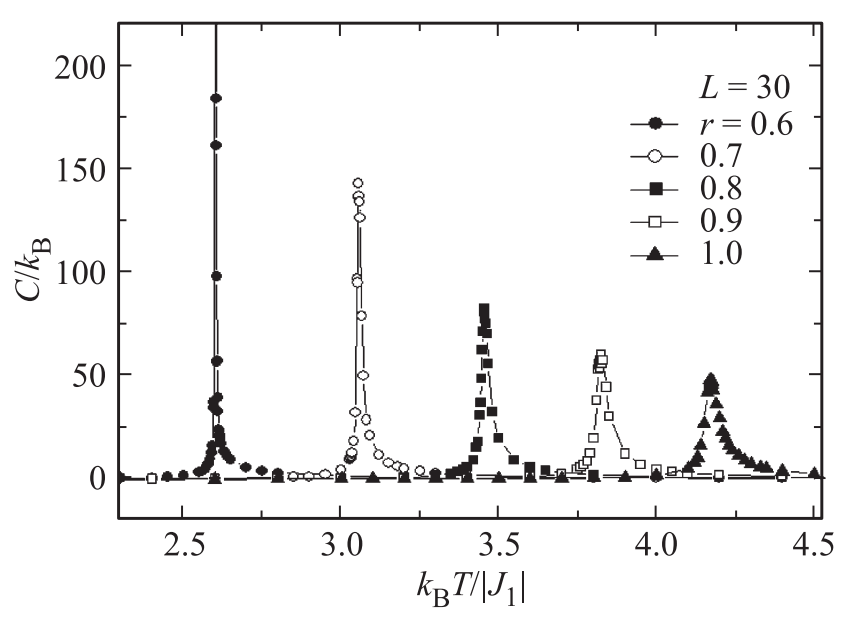

Рис. 2. Зависимость теплоемкости $C / k_{\mathrm{B}}$ от температуры $k_{\mathrm{B}} T /\left|J_{1}\right|$ для различных $r$.

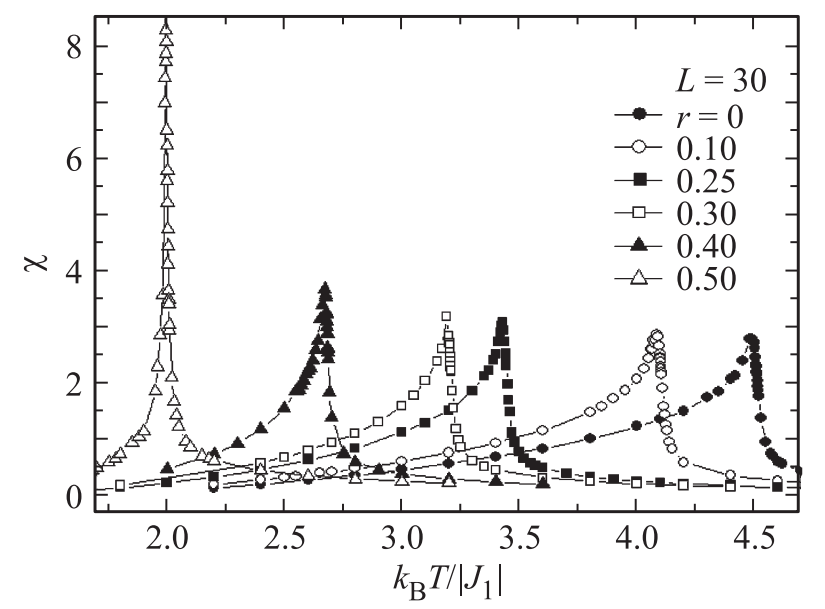

Рис. 3. Зависимость восприимчивости $\chi$ от температуры $k_{\mathrm{B}} T /\left|J_{1}\right|$ для различных $r$.

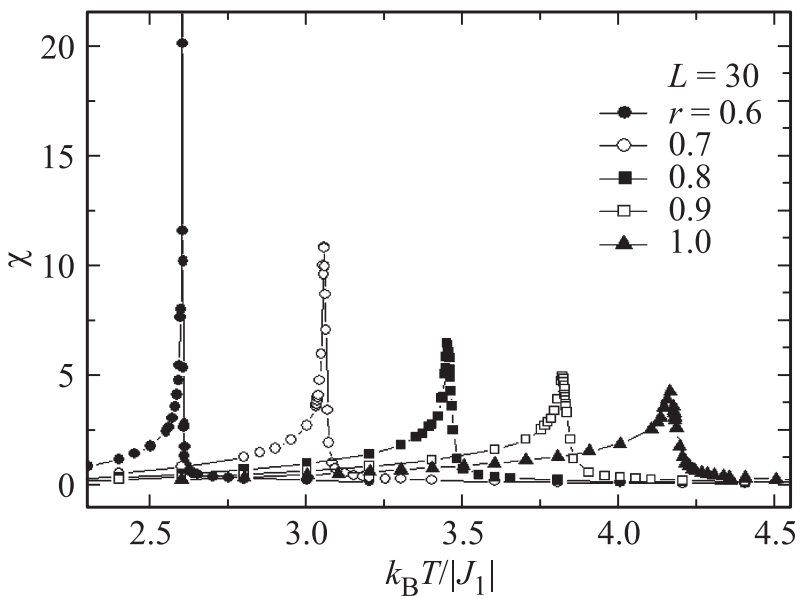

Рис. 4. Зависимость восприимчивости $\chi$ от температуры $k_{\mathrm{B}} T /\left|J_{1}\right|$ для различных $r$.

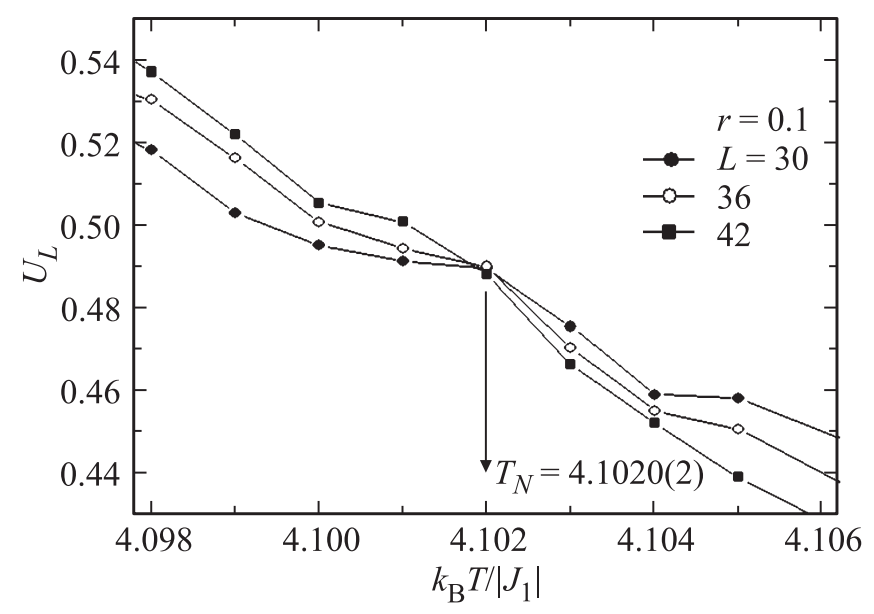

Рис. 5. Зависимости кумулянта Биндера $U_{L}$ от температуры $k_{\mathrm{B}} T /\left|J_{1}\right|$ для $r=0.1$ при различных $L$.

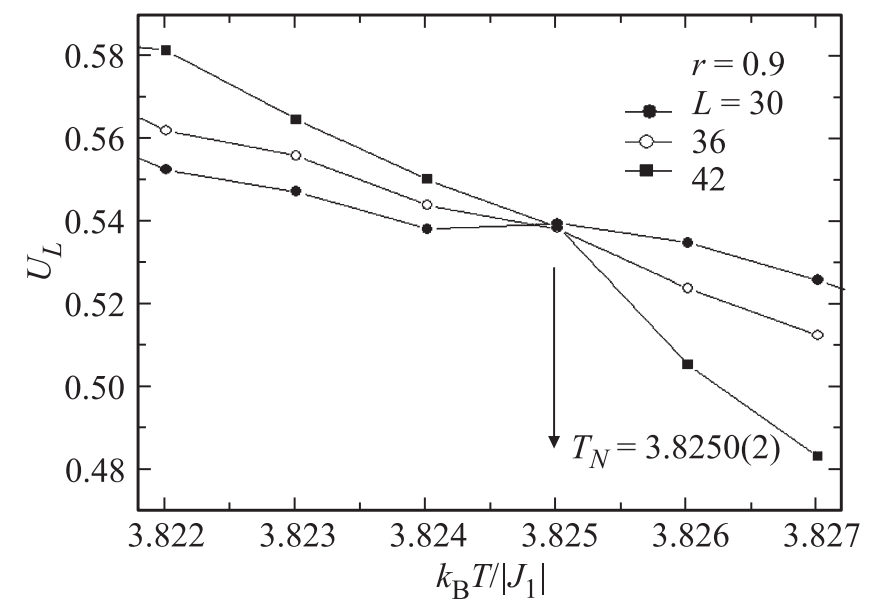

Рис. 6. Зависимости кумулянта Биндера $U_{L}$ от температуры $k_{\mathrm{B}} T /\left|J_{1}\right|$ для $r=0.9$ при различных $L$. 
Значения критических параметров для антиферромагнитной слоистой модели Изинга на кубической решетке

\begin{tabular}{c|l|l|l|l|l|l|c|c}
\hline$r$ & $k_{\mathrm{B}} T_{N} / J_{1} \mid$ & $v$ & $\alpha$ & $\beta$ & $\gamma$ & $\eta$ & $\alpha+2 \beta+\gamma=2$ \\
\hline $\begin{array}{c}\text { Нефрустрированная } \\
\text { модель Изинга }\end{array}$ & $4.5111(3)$ & $0.6305(25)$ & $0.108(9)$ & $0.3265(25)$ & $1.239(4)$ & $0.037(3)$ & \\
\hline 0.0 & $4.5110(2)$ & $0.630(5)$ & $0.110(5)$ & $0.320(5)$ & $1.241(5)$ & $0.03(1)$ & \\
0.1 & $4.1020(2)$ & $0.625(5)$ & $0.115(5)$ & $0.317(5)$ & $1.238(5)$ & $0.02(1)$ & 1.991 \\
0.2 & $3.4440(2)$ & $0.633(5)$ & $0.119(5)$ & $0.328(5)$ & $1.237(5)$ & $0.04(1)$ & 2.012 \\
0.3 & $3.2050(2)$ & $0.624(5)$ & $0.118(5)$ & $0.319(5)$ & $1.243(5)$ & $0.02(1)$ & 1.999 \\
0.4 & $2.6820(2)$ & $0.632(5)$ & $0.110(5)$ & $0.322(5)$ & $1.245(5)$ & $0.02(1)$ & 1.999 \\
0.9 & $3.8250(2)$ & $0.550(5)$ & $0.339(5)$ & $0.249(5)$ & $1.188(5)$ & $-0.14(1)$ & 2.025 \\
1.0 & $4.1730(2)$ & $0.549(5)$ & $0.330(5)$ & $0.245(5)$ & $1.190(5)$ & $-0.16(5)$ & 2 \\
$1.0[34]$ & $1.355(2)$ & $0.55(2)$ & $0.33(5)$ & - & - & $-0.28(6)$ & - & - \\
$1.0[35]$ & $1.347(1)$ & $0.56(2)$ & $0.32(2)$ & $0.25(2)$ & - & $-0.10(2)$ &
\end{tabular}

Для определения критической температуры $T_{N}$, нами использовался метод кумулянтов Биндера $U_{L}$ четвертого порядка [28]

$$
U_{L}=1-\frac{\left\langle m^{4}\right\rangle_{L}}{3\left\langle m^{2}\right\rangle_{L}^{2}}
$$

Согласно теории конечно-размерного скейлинга точка пересечения всех кривых $U_{L}(T)$ является критической точкой [28]. Выражение (8) позволяет определить критическую температуру $T_{N}$ с большой точностью.

На рис. 5 и 6 представлены характерные зависимости $U_{L}$ от температуры при $r=0.1$ и $r=0.9$ для разных значений $L$. Эти рисунки демонстрируют точность определения критической температуры. Видно, что в критической области наблюдаются четко выраженные точки пересечения $\left(T_{N}=4.1020(2)\right.$ для $r=0.1$ и $T_{N}=3.8250(2)$ для $r=0.9$; здесь и далее температура дана в единицах $\left.\left|J_{1}\right| / k_{\mathrm{B}}\right)$. Аналогичным образом были определены критические температуры и для остальных значений $r$, для которых согласно фазовой диаграмме полученной в работе [15], наблюдается ФП второго рода.

Для расчета статических критических индексов теплоемкости $\alpha$, параметра порядка $\beta$, восприимчивости $\gamma$ и радиуса корреляции $v$ применялись соотношения теории конечно-размерного скейлинга. Из теории конечноразмерного скейлинга следует, что в системе с размерами $L \times L \times L$ при $k_{\mathrm{B}} T /\left|J_{1}\right|=k_{\mathrm{B}} T_{N} /\left|J_{1}\right|$ и достаточно больших $L$ выполняются следующие условия [29-31]:

$$
\begin{aligned}
m & \sim L^{-\beta / v}, \\
\chi & \sim L^{\gamma / v}, \\
V_{i} & \sim L^{1 / v} g_{V_{i}},
\end{aligned}
$$

где $g_{V_{i}}$ - постоянная, а в качестве $V_{i}$ могут выступать

$$
V_{i}=\frac{\left\langle m^{i} E\right\rangle}{\left\langle m^{i}\right\rangle}-\langle E\rangle, \quad(i=1,2,3) .
$$

Эти выражения были нами использованы для определения $\beta, \gamma$ и $\nu$.
Для аппроксимации температурной зависимости теплоемкости от $L$ на практике, как правило, используется выражение [6]

$$
C_{\max }(L)=A_{1}-A_{2} L^{\alpha / v},
$$

где $A_{1}$ и $A_{2}$ - некоторые коэффициенты.

На рис. 7 и 8 в двойном логарифмическом масштабе представлены характерные зависимости параметров $V_{i}$ при $i=1,2,3$ от линейных размеров решетки $L$ для $r=0.1$ и $r=0.9$. Как видно из рисунков все точки на графиках в пределах погрешности хорошо ложатся на прямую. Зависимости на рисунках, проведенные в соответствии с методом наименьших квадратов, параллельны друг другу. Угол наклона прямой определяет значения $1 / v$. Вычисленное таким образом значение $v$ использовалось для определения критических индексов теплоемкости $\alpha$, параметра порядка $\beta$ и восприимчивости $\gamma$.

На рис. 9, 10, 11 и 12 в двойном логарифмическом масштабе представлены характерные зависимости магнитного параметра порядка $m$ и восприимчивости $\chi$ от линейных размеров решетки $L$ для $r=0.1$ и $r=0.9$. Все точки в пределах погрешности ложатся на прямые. Углы наклона этих прямых определяют значения $\beta / v$ и $\gamma / \nu$. По этой схеме были определены значения и для теплоемкости $\alpha / v$. На основе данных по $v$ вычислялись статические критические индексы $\alpha, \beta$ и $\gamma$.

Эта процедура использовалась для расчета критических индексов при значениях $r=1.0 ; 0.9 ; 0.4 ; 0.3 ; 0.2,0.1$ и 0.0. Все значения статических критических индексов, полученные таким образом, представлены в таблице.

Особо следует отметить процедуру, использованную нами для определения индекса Фишера $\eta$. Используя отношение между восприимчивостью $\chi$ и радиусом корреляции $\xi[32]$

$$
\chi \propto \xi^{\gamma / v},
$$

а также соотношение $\eta=2-\gamma / \nu$, связывающее индексы $\eta$ и $v$, мы получим

$$
\ln \left(\chi / \xi^{2}\right)=c-\eta \ln \xi
$$




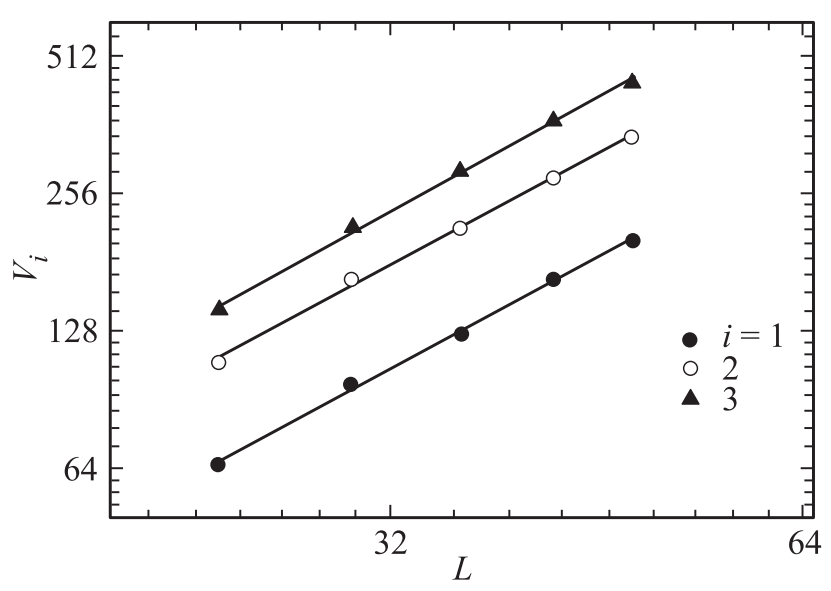

Рис. 7. Зависимость параметра $V_{i}$ от линейных размеров системы $L$ при $T=T_{N}$ для $r=0.1$.

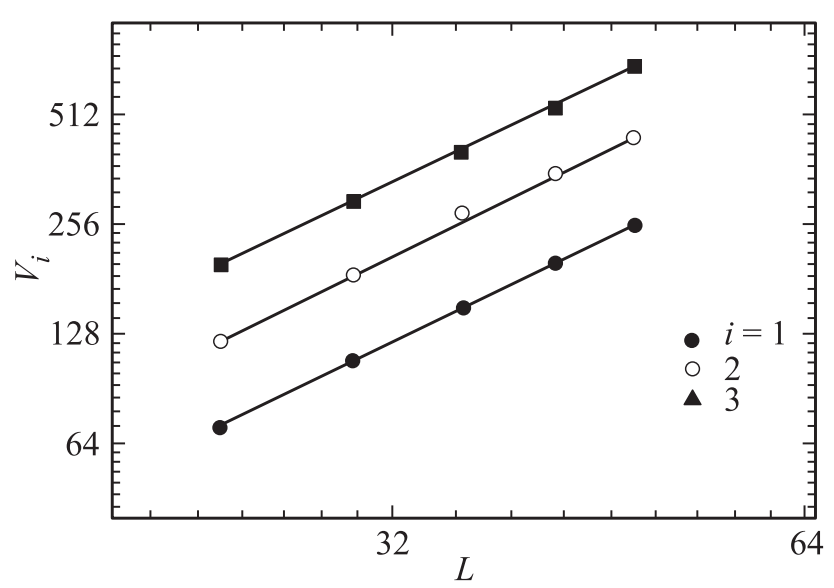

Рис. 8. Зависимость параметра $V_{i}$ от линейных размеров системы $L$ при $T=T_{N}$ для $r=0.9$.

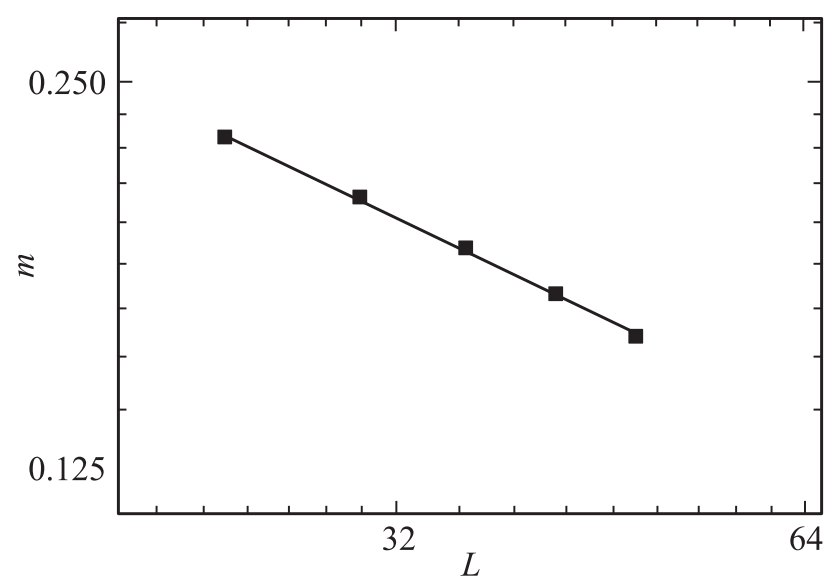

Рис. 9. Зависимость параметра порядка $m$ от линейных размеров системы $L$ при $T=T_{N}$ для $r=0.1$.

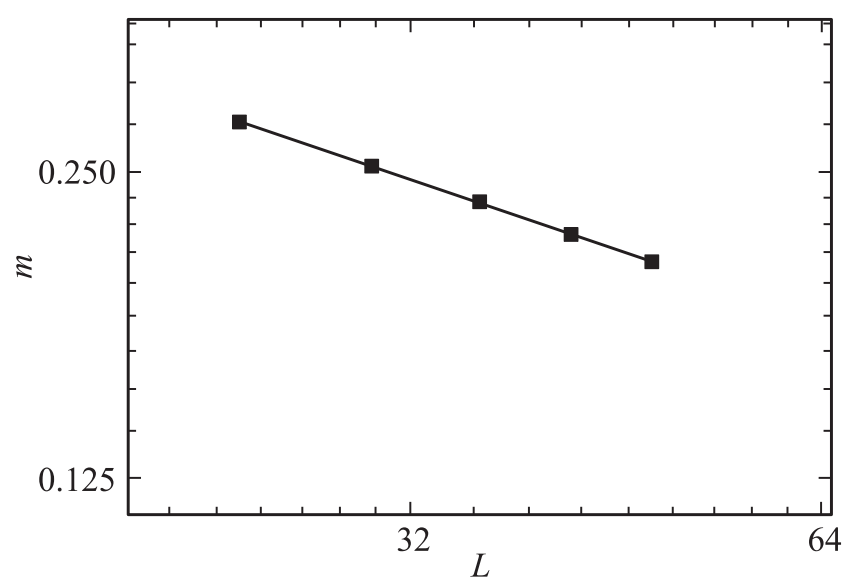

Рис. 10. Зависимость параметра порядка $m$ от линейных размеров системы $L$ при $T=T_{N}$ для $r=0.9$.

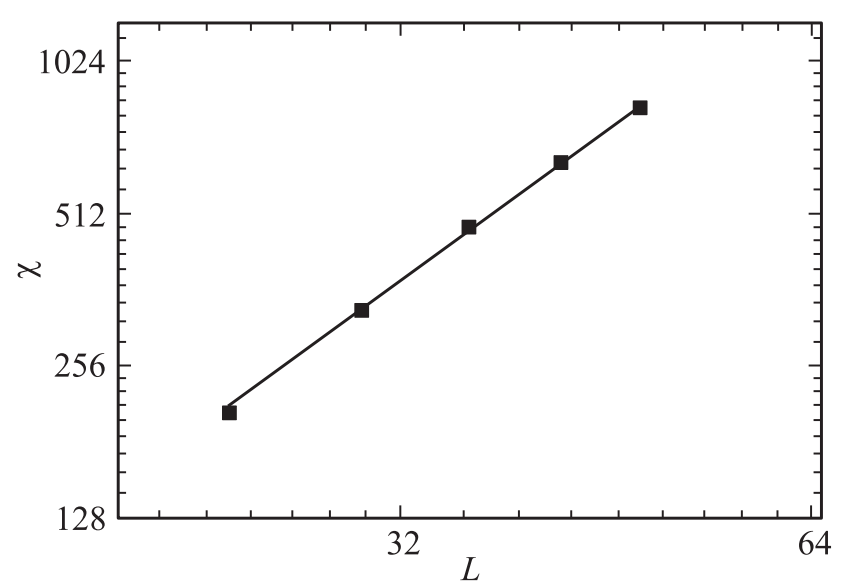

Рис. 11. Зависимость восприимчивости $\chi$ от линейных размеров системы $L$ при $T=T_{N}$ для $r=0.1$.

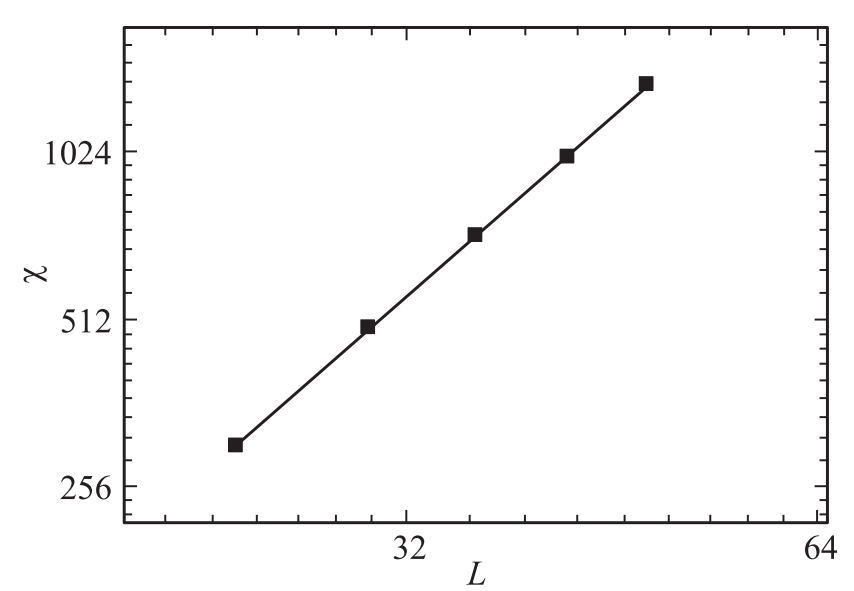

Рис. 12. Зависимость восприимчивости $\chi$ от линейных размеров системы $L$ при $T=T_{N}$ для $r=0.9$. 
где $c-$ некоторая константа. Для систем с конечными размерами $\xi=L$. Тогда при $k_{\mathrm{B}} T /\left|J_{1}\right|=k_{\mathrm{B}} T_{N} /\left|J_{1}\right|$ имеем

$$
\ln \left(\chi / L^{2}\right)=c-\eta \ln L \text {. }
$$

На основе выражения (16) было определено значение индекса Фишера $\eta$. Эти данные также представлены в таблице.

В настоящей статье, мы рассчитали критические параметры в интервале $0 \leq r \leq 0.4$ и для $r=0.9$ и $r=1.0$, где согласно результатам работы [15] исследуемая модель демонстрирует ФП второго рода. Для $r=0.5$ рассчитать критические параметры с допустимой погрешностью не удалось. Предполагаем, что это связано с тем, что в этой точке сосуществуют три различные фазы.

Сравнение численных значений критических индексов, полученные нами в данной работе с литературными данными показывает хорошее соответствие, причем многие критические параметры для данной модели для различных значений $r$ рассчитаны нами впервые.

Как видно в таблице, критическая температура $k_{\mathrm{B}} T_{N} /\left|J_{1}\right|$ уменьшается с увеличением величины взаимодействия следующих соседей вплоть до значения $r=0.4$. При дальнейшем увеличении $r$ критическая температура начинает расти. Все значения критических индексов, рассчитанные нами в интервале $0 \leq r \leq 0.4$, в пределах погрешности совпадают со значениями критических индексов трехмерной нефрустрированной модели Изинга [33]. Это свидетельствует о принадлежности данной модели в интервале $0 \leq r \leq 0.4$ к тому же классу универсальности критического поведения, что и нефрустрированная модель Изинга. Значения критических индексов, рассчитанные нами для $r=0.9$ и $r=1.0$, хорошо согласуются с теми, что получены в работах $[34,35]$ для полностью фрустрированной трехмерной модели Изинга на кубической решетке, но они сильно отличаются от данных, полученных нами для этой модели в интервале $0 \leq r \leq 0.4$. Можно предположить, что учет взаимодействий следующих ближайших соседей внутри слоев решетки для антиферромагнитной слоистой модели Изинга на кубической решетке приводит к тому же классу универсальности критического поведения, что и трехмерная полностью фрустрированная модель Изинга на кубической решетке.

\section{4. Заключение}

Исследование критического поведения антиферромагнитной слоистой модели Изинга на кубической решетке с учетом взаимодействия следующих ближайших соседей внутри слоев решетки выполнено с использованием высокоэффективного репличного алгоритма метода Монте-Карло. Определены значения критических температур и рассчитаны значения всех основных статических критических индексов в интервале $0 \leq r \leq 0.4$ и для $r=0.9$ и $r=1.0$. Установлены закономерности изменения критических параметров в рассмотренном интервале $r$. Обнаружено, что в интервале $0 \leq r \leq 0.4$ система проявляет универсальное критическое поведение. Показано, что в антиферромагнитной слоистой модели Изинга на кубической решетке с учетом взаимодействий следующих ближайших соседей внутри слоев решетки для значений $r=0.9$ и $r=1.0$ наблюдается другое критическое поведение.

\section{Список литературы}

[1] В.С. Доценко. УФН 165, 481 (1995).

[2] С.Е. Коршунов. УФН 176, 233 (2006).

[3] A. Malakis, P. Kalozoumis, N. Tyraskis. Eur. Phys. J. B 50, 63 (2006).

[4] D.P. Landau, K. Binder. Monte Carlo Simulations in Statistical Physics. Cambridge University Press, Cambridge (2000). P. 384.

[5] F.A. Kassan-Ogly, B.N. Filippov, A.K. Murtazaev, M.K. Ramazanov, M.K. Badiev. J. Mag. Mag. Mater. 324, 3418 (2012).

[6] А.К. Муртазаев, М.К. Рамазанов, М.К. Бадиев. ФНТ 37, 1258 (2011).

[7] K. Binder, D.P. Landau. Phys. Rev. B 21, 1941 (1980).

[8] F.A. Kassan-Ogly, A.K. Murtazaev, A.K. Zhuravlev, M.K. Ramazanov, A.I. Proshkin. J. Mag. Mag. Mater. 384, 247 (2015).

[9] А.К. Муртазаев, М.К. Рамазанов, Ф.А. Касан-Оглы, М.К. Бадиев. ЖЭТФ 144, 1239 (2013).

[10] A. Kalz, A. Honecker. Phys. Rev. B 86, 134410 (2012).

[11] S. Jin, A. Sen, A.W. Sandvik. Phys. Rev. Lett. 108, 045702 (2012).

[12] S. Jin, A. Sen, W. Guo, A.W. Sandvik. Phys. Rev. B 87, 144406 (2013).

[13] Р. Бэкстер. Точно решаемые модели в статистической механике. Мир, М. (1985). 488 с.

[14] М.К. Рамазанов, А.К. Муртазаев. Письма в ЖЭТФ 101, 793 (2015)

[15] М.К. Рамазанов, А.К. Муртазаев. Письма в ЖЭТФ 103, 522 (2016).

[16] A.R. dos Anjos, J.R. Viana, J.R. de Sousa, J.A. Plascak. Phys. Rev. E 76, 022103 (2007).

[17] D.R.O. Salmon, N. Crokidakis, A.M. Neto, T.I. Padilha, J.R. Viana, J.R. de Sousa. Int. J. Mod. Phys. B 27, 1350162 (2013).

[18] А.К. Муртазаев, М.К. Рамазанов, М.К. Бадиев. ФТТ 52, 1557 (2010).

[19] А.К. Муртазаев, М.К. Рамазанов. ФТТ 53, 1004 (2011).

[20] А.К. Муртазаев, М.К. Рамазанов, М.К. Бадиев. ЖЭТФ 142, 338 (2012).

[21] A.K. Murtazaev, M.K. Ramazanov, M.K. Badiev. Physica B 476, 1 (2015).

[22] M.K. Ramazanov, A.K. Murtazaev, M.A. Magomedov. Solid State Commun. 233, 35 (2016).

[23] М.К. Бадиев, А.К. Муртазаев, М.К. Рамазанов. ЖЭТФ 150, 722 (2016).

[24] А.К. Муртазаев, М.К. Рамазанов, Д.Р. Курбанова, М.К. Бадиев, Я.К. Абуев. ФТТ 59, 1082 (2017). 
[25] A. Mitsutake, Y. Sugita, Y. Okamoto. Biopolymers (Peptide Sci.) 60, 96 (2001).

[26] F. Wang, D.P. Landau. Phys. Rev. Lett. 86, 2015 (2001).

[27] K. Binder, J.-Sh. Wang. J. Stat. Phys. 55, 87 (1989).

[28] K. Binder, D.W. Heermann. Monte Carlo Simulation in Statistical Physics. Springer-Verlag, Berlin (1988); Nauka, M. (1995). P. 214.

[29] A.E. Ferdinand, M.E. Fisher. Phys. Rev. 185, 832 (1969).

[30] M.E. Fisher, M.N. Barber. Phys. Rev. Lett. 28, 1516 (1972).

[31] P. Peczak, A.M. Ferrenberg, D.P. Landau. Phys. Rev. B 43, 6087 (1991).

[32] Ch. Holm, W. Janke. Phys. Rev. B 48, 936 (1993).

[33] J.C. Le Guillou, J. Zinn-Justin. Phys. Rev. B 21, 3976 (1980).

[34] H.T. Diep, P. Lallemand, O. Nagai. J. Phys. C 181067 (1985).

[35] L.W. Bernardi, K. Hukushima, H. Takayama. J. Phys. A 32, 1787 (1999). 